\title{
Conduction band spectra in self-assembled InAs/GaAs dots: A comparison of effective mass and an eight-band approach
}

\author{
Hongtao Jiang and Jasprit Singh \\ Department of Electrical Engineering and Computer Science, The University of Michigan, Ann Arbor, \\ Michigan 48109-2122
}

(Received 21 May 1997; accepted for publication 30 September 1997)

\begin{abstract}
Strained epitaxy has been shown to produce high quality InAs/GaAs quantum dot structures by single step epitaxy. While effective mass-based approaches have been used for quantum structures, the nature of the strain and quantum confinement in self-assembled dots is such that this is not a good approximation. In this letter, we use an eight-band $\mathbf{k} \cdot \mathbf{p}$ formalism to find the electronic spectra in InAs/GaAs dots. The eight-band model shows that, in agreement with experiments, there are indeed several bound states in the conduction band well. Our results show that the simpler effective mass approaches cannot be used to quantitatively examine the physics of intersubband devices based on self-assembled quantum dots. Intersubband optical matrix elements and Coulomb blockade energy are also calculated in this letter. (C) 1997 American Institute of Physics.
\end{abstract}

[S0003-6951(97)01748-8]

Over the past decade a number of groups have shown that high strain epitaxy can be exploited to produce quantum dot structures by single step epitaxy. It is also known experimentally that these self-assembled dots have a pyramidal shape and have a highly complex strain tensor. The strong strain tensor causes a very large change in the effective bandgap and transitions in the quantum dots. For example, in InAs quantum dots on GaAs substrates, ${ }^{1-3}$ the ground state transition energy is $\sim 1.05 \mathrm{eV}$ even though the band gap of InAs is $0.4 \mathrm{eV}$. This large strain driven effect suggests that the electronic spectra of the structure should not be calculated by a simple effective mass approach. However, to simplify the problem calculations based on the effective mass picture have been reported in the literature. ${ }^{4-6}$ It is important to verify how accurate such a picture is.

In this letter we will address the following issue: What are the electron and hole spectra for InAs/GaAs quantum dots and what are the differences between the theoretical results based on an effective mass approximation (already reported by several groups in the literature) and an eightband $\mathbf{k} \cdot \mathbf{p}$ approximation in which the influence of remote bands is included?

The lattice mismatch between InAs and GaAs is $\sim 7 \%$ making the critical thickness $\sim 1.7$ monolayers. It is found experimentally ${ }^{1}$ that once the thickness of InAs exceeds a few monolayers, InAs islands are formed on top of a wetting layer. For growth along [001] direction these islands are small pyramidal shaped with a square base in the (001) plane. The limiting planes of the islands vary for different experiments. ${ }^{1,5}$ For small base angle, it is reasonable to assume the strain is biaxial strain over all the dot area and this was done by Ref. 5. But if the base angle is not small, the strain distribution is expected to be quite different from the biaxial strain and this is verified by calculations done in Ref. 4 based on the use of elastic continuum theory.

We use the valence force field (VFF) model by Keating ${ }^{7}$ and Martin $^{8}$ to calculate the strain distribution. VFF model is a microscopic theory which includes the bond stretching and bond bending and avoids the potential failure of elastic con- tinuum theory in the atomically thin limit. This model has been used by Ref. 6 to calculate the strain distribution in QDs, and our results show much the same as Ref. 6 .

The problem of band structure in semiconductor quantum structures has usually been solved by decoupling the conduction and valence band problems. The conduction band problem is typically solved using a scalar effective mass approach while the valence band problem is solved using a k. p approach which includes the heavy hole and light hole coupling. This decoupling is possible because in problems typically encountered (for example, quantum wells and wires or even quantum dots with small strain), there is minimal remote band influence on the conduction band. However, in the self-assembled quantum dot this is not the case. We note the following: (i) the band gap of bulk InAs is $0.4 \mathrm{eV}$, while the effective gap of the dot is $\sim 1.0 \mathrm{eV}$; (ii) the nature of the strain tensor is such that there is a strong spatial variation in strain; (iii) the strain components are very large and the resultant splittings in the bands are comparable to the interband separations in the bulk material. All these considerations suggest that the simple decoupled conduction-valence band picture and the effective mass description may not be adequate.

We use the eight-band $\mathbf{k} \cdot \mathbf{p}$ description where the influence of remote bands on the conduction and valence band states is included. The strain effect is included via deformation potential theory. ${ }^{9}$ The four-band Kohn-Luttinger parameters can be converted to eight-band parameters according to Refs. 9 and 10. The eight-band Hamiltonian includes the effects of the valence states and the conduction states of the deformed structure. Thus there is no need to a priori assume an effective mass for any of the bands.

Both the conduction band states and valence band states can be derived from eight-band Hamiltonian. We use the finite difference method to solve this equation numerically. The distance between two grid points is chosen to be $5.65 \AA$ (GaAs lattice constant) in the $z$ direction and two times this distance in the $x, y$ plane. Harmonic averaging ${ }^{11}$ of the parameters is used in the interface region and the eigenvalue 

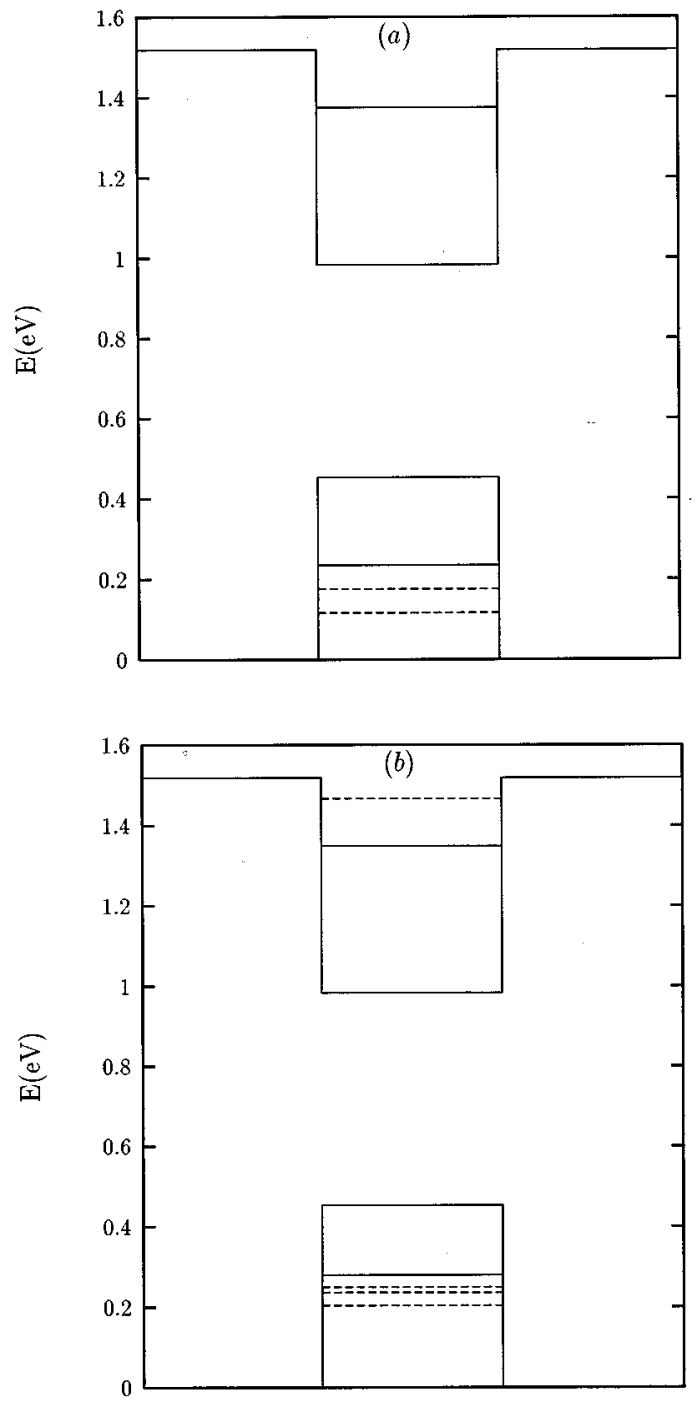

FIG. 1. Electronic spectrum for a InAs/GaAs dot with base width $113 \AA$ and height $56.5 \AA$ A. Solid lines represents states that can be observed in PL spectra and dashed lines represents states that cannot be observed in PL spectra. (a) $m^{*}=0.023 m_{0}$ for conduction band and $m_{h h z}=0.5263 m_{0}$, $m_{h h \perp}=0.0356 m_{0}$ for heavy hole. (b) $m^{*}=0.04 m_{0}$ for conduction band and a four-band $\mathbf{k} \cdot \mathbf{p}$ model for valence band.

problem is solved by the implicitly restarted Arnoldi/ Lanczos methods. ${ }^{12}$

In order to show the differences between eight-band $\mathbf{k} \cdot \mathbf{p}$ model and effective mass model, we will calculate the band structure of a quantum dot using different models. The dot has a base width $113 \AA$ and a height $56.5 \AA$ with limiting plane in (101). The wetting layer is 1.5 monolayer. The dots of similar size have been examined in Refs. 2 and 4 and in Ref. 6 using effective mass model in order to understand the experimental results.

In Ref. 4 the conduction band states have been addressed using an effective mass description with a mass of $0.023 m_{0}$. The valence band states are also calculated using a decoupled heavy hole light hole picture. The results shown in Fig. 1(a) use this picture. This description gives a single bound state in the conduction band well. Recognizing that the effective mass should correspond to a larger gap material, results given in Ref. 6 use the effective mass $0.04 m_{0}$ instead of

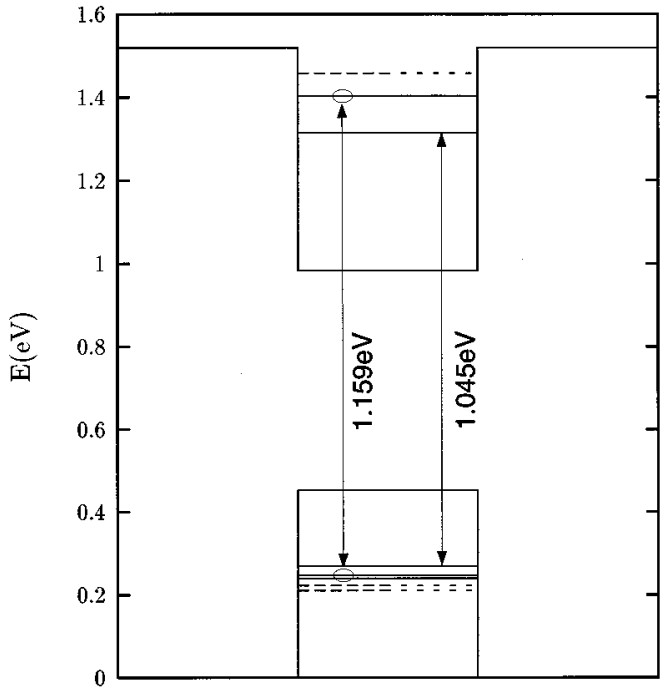

FIG. 2. Electronic spectrum for a InAs/GaAs dot with base width $113 \AA$ and height $56.5 \AA$ calculated using the eight-band $\mathbf{k} \cdot \mathbf{p}$ model described in the text.

$0.023 m_{0}$. The spectra in Fig. 1(b) are calculated using the effective mass $0.04 m_{0}$ for conduction band and four-band model for valence bands. The use of a larger effective mass gives two bound levels in the conduction band. However, as noted in Ref. 6 a more sophisticated Hamiltonian is needed to shed more light on the excited states.

In Fig. 2 we show the results obtained from the eightband model. We see that there are a number of excited states in the conduction band. Such richness of spectra is seen qualitatively in experiments. From our calculations we find that the first and second transitions observed in Ref. 2 are due to the ground electronic states to ground hole states and excited electronic states to excited hole states. Unlike the case shown in Refs. 2 and 4 the second transition is not due to the ground electronic states to excited hole states. An interesting feature of the calculated spectra is the near fourfold degeneracy of the second excited states. These states have an exact double degeneracy due to time-reflection symmetry and are almost degenerate due to different excitation directions [110] or [110]. The second excited electronic state is 54 $\mathrm{meV}$ higher than the first excited states. There is considerable mixture of wetting layer states in this state because it is less confined. The direction of excitation is in the $z$ direction. It should be noted for a dot with smaller base angle (such as the case that the limiting plane is (102)) the second excited direction is still in the $x, y$ plane.

We also calculated the Coulomb blockade energy according to first perturbation theory. The Coulomb energy is $32 \mathrm{meV}$ for the case both of the electrons are in the ground orbital states. It changes to $26 \mathrm{meV}$ for the case one is in the first excited states and another is in the ground states. Here InAs dielectric constant is used. It maybe pointed out that the Coulomb blockade energy between ground states and excited states is quite sensitive to the shape of the dots.

As found by Ref. 6, we also find many confined hole states. We find that the splitting between the ground and excited states can change from 10 to $30 \mathrm{meV}$ for different Kohn-Luttinger parameters. There are no wavefunction overlap between second excited hole states and second ex- 

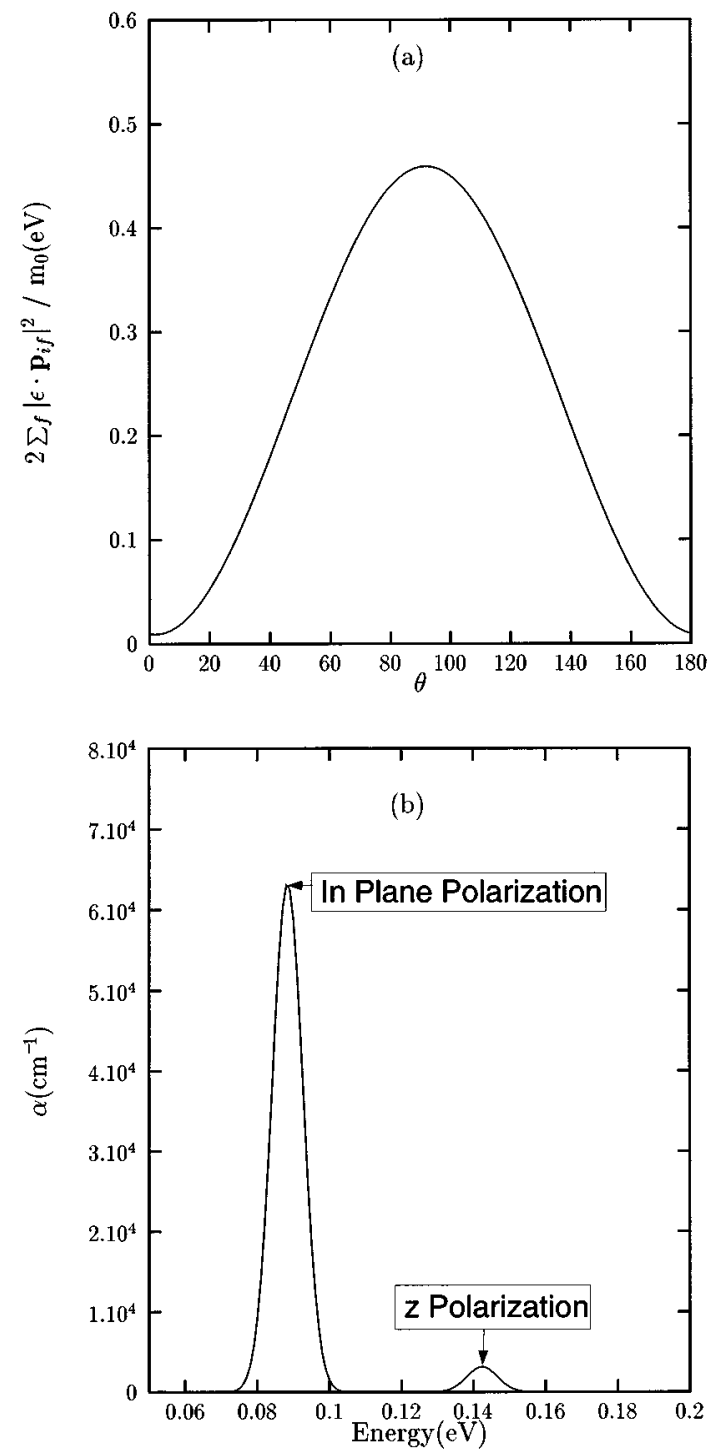

FIG. 3. (a) The dependence of inter-subband matrix element between ground states and first excited states on the polarization of the light. $\theta$ is the polar angle. (b) Intersubband absorption spectra for the InAs/GaAs dot with base width $113 \AA$ and height $56.5 \AA$.

cited electron states due to different excitation directions.

The dependence of intersubband optical transition matrix between ground electronic states and first excited states on polarization is shown in Fig. 3(a). The dominant contribution is from Block function part (i.e., the $p$ mixture in the conduction band) instead of envelope function part. If only envelope part is included, the value is two orders of magnitude smaller. This corresponds to use effective electron mass instead of free electron mass in the intersubband transition as proved by Ref. 13. There is no polarization dependence in the plane of QDs due to almost degeneracy of states in the two different excitation directions. The matrix element is very small for the polarization in the growth direction.

In Fig. 3(b) we show results for intersubband optical absorption assuming that the ground state is occupied by a single electron in a dot (through doping, as in an intersubband detector). The absorption coefficient (which goes as inverse of the transition linewidth) has a strong coupling with in-plane polarized light for the ground states to the first excited states transition. It has a strong coupling with $z$-polarized light for the ground states to the second excited states transition. Here the FWHM $10 \mathrm{meV}$ is assumed. Note that the absorption coefficient is approximately

$$
\alpha \sim \frac{6.3 \times 10^{5}}{\sigma} \mathrm{cm}^{-1}
$$

where $\sigma$ is the linewidth in meV. The above value is calculated for a single dot. For a sample containing array of dots, it has to be scaled with filling factor of the dots. As a result, the uniformity in dot sizes and the density of dots are quite critical.

In summary, we have calculated the strain distribution in a typical self-assembled InAs/GaAs dot using the valence force field method. The electronic spectra has also been studied using an eight-band $\mathbf{k} \cdot \mathbf{p}$ model. Our results show that the effective mass approach for the conduction band gives significant errors and also suffers from the problem that there is no nonarbitrary choice for the effective mass. The eight-band model gives a series of bound states in the quantum dot conduction band in contrast to the effective mass approach which only gives one or two bound states. The experimental spectra show the presence of several electronic states in the conduction band-a result that is explained by the eightband model

One of the authors (H. Jiang) is grateful to R. Jambunathan for helpful suggestions. This work was funded by National Science Foundation Grant No. ECS9628973 and by ARPA under MDA972-94-1-0004.

${ }^{1}$ M. Grundmann, J. Christen, N. N. Ledentsov, J. Böhrer, D. Bimberg, S. S. Ruvimov, P. Werner, U. Richter, U. Gösele, J. Heydenreich, V. M. Ustinov, A. Yu. Egorov, A. E. Zhukov, P. S. Kop'ev, and Zh. I. Alfenov, Phys. Rev. Lett. 74, 4043 (1995).

${ }^{2}$ F. Adler, M. Geiger, A. Bauknecht, F. Scholz, H. Schweizer, M. H. Pilkuhn, B. Ohnesorge, and A. Forchel, J. Appl. Phys. 80, 4019 (1996).

${ }^{3}$ K. H. Schmidt, G. Medeiros-Ribeiro, M. Oestreich, and P. M. Petroff, Phys. Rev. B 54, 11346 (1996).

${ }^{4}$ M. Grundmann, N. N. Ledentsov, O. Stier, D. Bimberg, V. M. Ustinov, P. S. Kopev, and Zh. I. Alferov, Appl. Phys. Lett. 68, 979 (1996); M. Grundmann, O. Stier, and D. Bimberg, Phys. Rev. B 52, 11969 (1995).

${ }^{5}$ J.-Y. Marzin and G. Bastard, Solid State Commun. 92, 437 (1994).

${ }^{6}$ M. A. Cusack, P. R. Briddon, and M. Jaros, Phys. Rev. B 54, R2300 (1996)

${ }^{7}$ P. N. Keating, Phys. Rev. 145, 637 (1966).

${ }^{8}$ R. M. Martin, Phys. Rev. B 1, 4005 (1969).

${ }^{9}$ F. H. Pollak, Semicond. Semimet. 32, 17 (1990); J. Singh, Physics of Semiconductors and Their Heterostructures (McGraw-Hill, New York, 1993).

${ }^{10}$ G. L. Bir and G. E. Pikus, Symmetry and Strain-Induced Effects in Semiconductors (Wiley, New York, 1974).

${ }^{11}$ T. L. Li and K. J. Kuhn, J. Comp. Physiol. 110, 292 (1994).

${ }^{12}$ R. Lehoucq, D. C. Sorensen, and C. Yang, ARPACK Users Guide: Solution of Large Scale Eigenvalue Problems by Implicitly Restarted Arnoldi Methods, 1997 (submitted to SIAM Publications).

${ }^{13}$ R. Q. Yang, Phys. Rev. B 52, 11958 (1995). 